\title{
The TP53 intron 6 G13964C Polymorphism and Risk of Thyroid and Breast Cancer Development in the Iranian Azeri Population
}

\author{
Roghayeh Dehghan ${ }^{1}$, Mohammad Ali Hosseinpour Feizi ${ }^{1}$, Nasser Pouladi ${ }^{1,2 *}$, \\ Mina Adampourezare ${ }^{1}$, Davoud Farajzadeh ${ }^{2}$
}

\begin{abstract}
Background: TP53 mutations are the most common genetic alterations in human cancers. There are also several polymorphisms in both exons and introns of TP53 that may influence its anti-tumor functions and increase the risk of cancer development. Associations of the TP53 intron $6 \mathrm{G13964C}$ polymorphism with increased risk of development of several cancers have been investigated in numerous studies, but the results were controversial and conflicting. In this study, we aimed to investigate the probable association of this polymorphism with risk of both thyroid and breast cancers among the Iranian-Azeri population. Materials and Methods: We performed two separate case control studies on associations of the intron 6 polymorphism with two different kinds of cancer. In one case-control study, a total of 75 patients with thyroid carcinoma and 180 controls were analyzed and the other study included 170 patients with breast cancer and 135 healthy women. The intron 6 genotype was determined by RFLP-PCR and the SPSS 16 program was applied for data analysis. Results: For thyroid cancer, the frequencies of GG genotype were $96.0 \%$ in patients and $93.3 \%$ in controls. The GC genotype had a frequency of $4.0 \%$ in patients and $6.7 \%$ in controls. In the study on breast cancer, the frequency of GG and GC genotypes in patients were $95.3 \%$ and $4.7 \%$, respectively. In breast related control group, the frequency of GG genotype was $93.3 \%$ and the frequency of GC genotype was $6.7 \%$. None of the cases and controls had the CC genotype. Conclusions: There was no significant association between the TP53 intron 6 G13964C polymorphism and risk of development of both thyroid and breast cancer in Iranian-Azeri patients.
\end{abstract}

Keywords: Thyroid - breast - cancer - P53 tumor suppressor protein - polymorphism - molecular marker

Asian Pac J Cancer Prev, 16 (7), 3073-3077

\section{Introduction}

TP53 gene encodes a crucial transcription factor that is activated in response to various stresses such as hypoxia, DNA damage and oncogene activation and protects cells from neoplastic transformation through cell cycle arrest or apoptosis (Stegh et al., 2012; Pouladi et al., 2013). This tumor suppressor protein is mutated in about half of the cancers and it seems that the other half is developed by alterations in P53 related genes or pathways (MachadoSilva et al., 2010; Sedaie-Bonab et al., 2014). The TP53 status of a tumor could significantly affect the prognosis of patients and serve as an important diagnostic marker to distinguish the different variants of a tumor (Radha et al., 2014; Shin et al., 2014).

Breast cancer is the most common cancer of women in the world. Public health schemes in the developed countries have led to the stabilization or even decrease in the incidence of breast cancer, nevertheless in most developing countries, the incidence rate is certainly rising
(Pathy et al., 2011; American cancer society, 2013; Pouladi et al., 2014). Molecular changes, including the P53 gene mutations, are related to increased risk of breast cancer development. In females with p53 mutations, the breast cancer is the most common malignancy and in comparison with general population, p53 mutation carriers have from an 18- to 60-fold increased risk for early onset breast cancer (diagnosed before the age of 45) (Olivier et al., 2003; Walsh et al., 2006).

Unlike breast, the thyroid cancer incidence is low and it is estimated that it only comprises approximately $1 \%$ of all malignancies. Nevertheless, thyroid cancer is the most prevalent malignancy of endocrine system and in recent decades; epidemiological studies have been shown a steady increase in its incidence in many countries (Sipos et al., 2010; Akbari et al., 2011). Various genetic alterations have been observed in thyroid tumors that TP53 inactivation is among them (Legakis et al., 2009). Several studies have been cleared that TP53 mutations are mostly common in anaplastic and poorly differentiated carcinomas and 
rarely found in benign and differentiated tumors. In fact, it seems that P53 mutations occur as secondary alterations and leads to more dedifferentiation and aggressiveness of differentiated carcinomas (Parameswaran et al., 2010; Montagna et al., 2011; Dehghan et al., 2014).

Besides mutations, there are some important polymorphisms in p53 that their association with the risk of cancer development has been reported in some studies; however the results have been conflicting (Whibley et al., 2009). Intronic mutations and polymorphisms can occur within regulatory sequences like promoters, enhancers, silencers and regulatory miRNA or affect gene expression by altering RNA splicing (Malkinson et al., 1994). G13964C substitution (rs 17880604) is one of the wellstudied intronic polymorphisms of P53 that is not within splice site consensus sequences or enhancers. However, some studies have been suggested that $13964 \mathrm{G}$ variant contributes to the stability of P53 mRNA and protein (Lehman et al., 2000). The association of the 13964C variant has been investigated in increased risk of various cancers like breast (Trifa et al., 2010; Alawadi et al., 2011; Surekha et al., 2011), ovarian (Liu et al., 2004) and colon (Buyru et al., 2005), but the results have not been completely consistent.

In this study, we have analyzed the association of P53 G13964C polymorphism with the increased risk of thyroid and breast cancers development among IranianAzeri population.

\section{Materials and Methods}

\section{Sample collection}

A total of 75 Iranian-Azeri patients with thyroid cancer and 170 ones with breast cancer have been participated in this study with their consent. All of the patients had undergone thyroidectomy or mastectomy surgery at Imam Reza or Noor-E-Nejat hospitals in Tabriz, Iran between 2010 and 2013. The blood samples also were obtained from 180 Azeri controls with no history of cancer among their relatives. This group included 135 women and 45 men that all of them were analyzed as controls in relation to thyroid cancer but only the women participated as control group in association study of G13964C polymorphism with breast cancer.

\section{Genotyping of P53 polymorphism:}

The proteinase $\mathrm{K}$ and salting out method were used to extract DNA from both blood and tissue samples. The genotype Intron 6 G13964C polymorphism was determined using PCR-RFLP technique. For this purpose, the polymorphism containing fragment was amplified using the following primers:

\section{5'-GCCCTCCCCTGCTTGCC-3' \\ 5'-CCGCCCATGCAGGAACT-3'}

Each PCR reaction was performed in a total volume of $25 \mu \mathrm{l}$ containing 1 to $2 \mu \mathrm{l}$ DNA (with an average concentration of $200 \mathrm{ng}$ ), $2.5 \mathrm{ml}$ of $10 \mathrm{X}$ PCR buffer, $1 \mu \mathrm{l}$ $\mathrm{MgCl} 2,0.5 \mu \mathrm{ldNTP}, 0.75 \mu \mathrm{l}$ of each forward and reverse primers $(10 \mathrm{pM})$ and $0.15 \mu \mathrm{l}$ of $\mathrm{Taq}$ DNA polymerase enzyme. The 131 bp PCR amplicon was digested with 2 units of Hin6I (Fermentase) enzyme at $37^{\circ} \mathrm{C}$ overnight and RFLP products electrophoresed on a $3 \%$ agarose gel. Digestion resulted to two 98 and $33 \mathrm{bp}$ fragments for the $\mathrm{G}$ allele and a single $131 \mathrm{bp}$ fragment for the $\mathrm{C}$ allele (Figure 1).

\section{Statistical analysis}

The online HWE calculator, http://www.oege.org/ software/hardy-weinberg.html, was applied to assess Hardy- Weinberg equilibrium based on a pearson's chisquare test for both control and case groups. Pearson's chisquare test or Fisher's exact test (if there was any cell with an expected count less than 5) were used to analyze the relationship between the polymorphism and increased risk of neoplasia development, as well as clinicopathological characteristics. Odds ratios with $95 \%$ confidence intervals were calculated to determine the relationship between the each genotype and allele with thyroid neoplasia development. pearson's chi-square or Fisher's exact tests and Odds ratio calculation were performed using SPSS 16 software and the Javastat online statistics package (http://statpages.org/ctab2x2.html). The p value $<0.05$ was considered significant.

\section{Results}

\section{Thyroid cancer}

To investigate the association of P53 G13964C with increased risk of thyroid cancer, we genotyped a total of 75 thyroid cancer patients with the mean age of $38.45 \pm 12.96$ and 180 healthy subjects with the mean age of $46.53 \pm 12.19 .73 .3 \%$ of cases were female and $26.7 \%$ were male. Among 180 controls, $75.0 \%$ were female and $25.0 \%$ were male. According to clinicopathological information, among thyroid patients, 66 patients presented papillary thyroid carcinoma, 4 patients had follicular thyroid carcinoma and 2 had medullary thyroid carcinoma. Based on TNM staging, the tumor stage in $88 \%$ of patients was I or II and $12 \%$ had stage III tumor.

Pearson chi-square test was performed to compare observed genotype and allele frequencies with those that are expected in a population with Hardy-Weinberg equilibrium. The frequencies of genotypes and alleles did not show a deviation from Hardy-Weinberg equilibrium in both patient and control groups (Table 1). The frequencies of GG genotype were $96.0 \%$ in patients and $93.3 \%$ in controls. The GC genotype had a frequency of $4.0 \%$ in cases and $6.7 \%$ in controls. The CC

Table 1. Allele and Genotype Frequencies of p53 Intron 6 Polymorphisms in Thyroid Cancer Patients and Controls

\begin{tabular}{|c|c|c|c|c|}
\hline & Controls & $\begin{array}{l}\text { Thyroid } \\
\text { patients }\end{array}$ & $\begin{array}{c}\text { Odds ratio } \\
(95 \% \mathrm{CI})\end{array}$ & $P$ value \\
\hline \multicolumn{5}{|c|}{ Intron $6 \mathrm{G} 13964 \mathrm{C}$ polymorphisms } \\
\hline GG & $168(93.3 \%)$ & $72(96.0 \%)$ & reference & - \\
\hline GC & $12(6.7 \%)$ & $3(4.0 \%)$ & $0.583(0.126-2.313)$ & 0.564 \\
\hline $\mathrm{CC}$ & 0 & 0 & - & - \\
\hline G & $348(96.7 \%)$ & $147(98.0 \%)$ & reference & - \\
\hline $\mathrm{C}$ & $12(3.3 \%)$ & $3(2.0 \%)$ & $0.592(0.131-2.292)$ & 0.57 \\
\hline HWE & $E^{*} \quad 0.643$ & 1 & - & - \\
\hline
\end{tabular}

*Hardy-Weinberg Equilibrium $P$ value 
Table 2. Allele and Genotype Frequencies of p53 intron 6 Polymorphisms in Breast Cancer Patients and Controls

\begin{tabular}{lcccc}
\hline \multicolumn{1}{c}{ Controls } & $\begin{array}{c}\text { Breast } \\
\text { patients }\end{array}$ & $\begin{array}{c}\text { Odds ratio } \\
(95 \% \mathrm{CI})\end{array}$ & $P$ value \\
\hline Intron 6 G13964C & polymorphisms \\
GG & $126(93.3 \%)$ & $162(95.3 \%)$ & reference & - \\
GC & $9(6.7 \%)$ & $8(4.7 \%)$ & $0.691(0.235-2.021)$ & 0.458 \\
CC & 0 & 0 & - & - \\
G & $261(96.7 \%)$ & $332(97.6 \%)$ & reference & - \\
C & $9(3.3 \%)$ & $8(2.4 \%)$ & $0.699(0.242-2.005)$ & 0.465 \\
HWE* & 0.688 & 0.753 & - & - \\
\hline
\end{tabular}

*Hardy-Weinberg Equilibrium P value

Table 3. Association of p53 Intron 6 Polymorphism with Clinicopathological Characteristics among Patients with Thyroid Cancer

\begin{tabular}{lcccc}
\hline & GG & GC & Total & $P$ value \\
\hline Age & & & & 1 \\
$\leq 0$ & $45(95.7 \%)$ & $2(4.3 \%)$ & 47 & \\
$<40$ & $27(96.4 \%)$ & $1(3.7 \%)$ & 28 & \\
Gender & & & & 0.565 \\
$\quad$ Female & $52(94.5)$ & $3(5.5)$ & 55 & \\
Male & $20(100 \%)$ & 0 & 20 & \\
Tumor type & & & & 0.258 \\
PTC* & $66(97.1 \%)$ & $2(2.9 \%)$ & 68 & \\
FTC** & $4(80.0 \%)$ & $1(20.0 \%)$ & 5 & \\
MTC*** & $2(100 \%)$ & 0 & 2 & \\
Iymph node metastasis & & & 1 \\
Positive & $36(94.7 \%)$ & $2(3.3 \%)$ & 38 & \\
Negative & $36(97.3 \%)$ & $1(3.7 \%)$ & 37 & \\
Tumor stage & & & & 0.999 \\
I & $57(95.0 \%)$ & $3(5.0 \%)$ & 60 & \\
II & $6(100 \%)$ & 0 & 6 & \\
III & $9(100 \%)$ & 0 & 9 & \\
Papillary Thyr & & &
\end{tabular}

*Papillary Thyroid Carcinoma, **Follicular Thyroid Carcinoma, ***Medullary Thyroid Carcinoma

genotype was not observed in any group. There was no significant association between TP53 intron 6 G13964C polymorphism and increased risk of thyroid neoplasia development $(\mathrm{p}>0.05)$. Also, No association was observed between intron 6 polymorphism and any clinicopathological characteristic (Table 3).

\section{Breast cancer}

Among breast patients, 158 people had invasive ductal carcinoma, 9 had invasive lobular carcinoma and 3 of them presented with ductal carcinoma in situ. The mean age of breast patients was $46.26 \pm 11.04$. The tumor stage in $48.2 \%$ of patients was I or II and $50.0 \%$ had stage III or IV tumor. The frequency of stage 0 was only $1.8 \%$. The other clinicopathological information are given in table 4 . The frequency of GG and GC genotypes in patients were $95.3 \%$ and $4.7 \%$, respectively. In breast related control group, the frequency of GG genotype was $93.3 \%$ and the frequency of GC genotype was $6.7 \%$. None of the cases and controls had the CC genotype. Like as thyroid cancer, no association has been found between intron $6 \mathrm{G} 13964 \mathrm{C}$ polymorphism and the risk of breast cancer development as well as clinicopathological characteristics.
Table 4. Association of p53 Intron 6 Polymorphism with Clinicopathological Characteristics among Patients with Breast Cancer

\begin{tabular}{lcccc}
\hline \multicolumn{1}{c}{ GG } & GC & Total & $P$ value \\
\hline Age & & & & 0.725 \\
$\leq 40$ & $81(94.2 \%)$ & $5(5.8 \%)$ & 86 & \\
$<40$ & $81(96.4 \%)$ & $3(3.6 \%)$ & 84 & \\
Tumor type & & & & 0.188 \\
IDC* & $151(95.6 \%)$ & $7(4.4 \%)$ & 158 & \\
ILC** & $9(100 \%)$ & 0 & 9 & \\
DCIS*** & $2(96.7 \%)$ & $1(3.3 \%)$ & 3 & \\
Tumor size & & & & 1 \\
$\leq 3.5$ & $80(95.2 \%)$ & $4(473 \%)$ & 84 & \\
$<3.5$ & $82(95.3 \%)$ & $4(4.7 \%)$ & 86 & \\
Iymph node metastasis & & & 1 \\
Positive & $100(95.2 \%)$ & $5(4.8 \%)$ & 105 & \\
Negative & $62(95.4 \%)$ & $3(4.6 \%)$ & 65 & \\
Tumor stage & & & & 0.154 \\
stage 0 & $2(66.7 \%)$ & $1(33.3 \%)$ & 3 & \\
Early(I\&II) & $78(95.1 \%)$ & $4(4.9 \%)$ & 82 & \\
Late (III\&IV) & $72(96.5 \%)$ & $3(3.5 \%)$ & 75 & \\
*Invasive Ductal Carcinoma,**Invasive Lobular Carcinoma, ${ }^{* * *}$ Ductal \\
Carcinoma In Situ
\end{tabular}

\section{Discussion}

For the first time, Buller and et al found the 13964C variant of intron 6 in three ovarian cancer patients with a history of metachronous breast cancer. They considered it as a polymorphism, because it was located in the intronic region and did not show an association with TP53 overexpression in tumor samples obtained from patients carrying C allele (Buller et al., 1995). Later, Lehman and et al found this variant in $7.1 \%$ of 42 American patients with familial breast cancer, but not in any of 171 sporadic breast cancer cases. In their in vitro studies on lymphoblastoid cell lines, derived from patients with $13964 \mathrm{C}$ variant, it was revealed that $\mathrm{G}$ to $\mathrm{C}$ substitution in intron 6 is associated with a long-term cell survival after DNA damage and reduced apoptosis after chemotherapy (Lehman et al., 2000). In addition, strong nuclear P53 immunoreactivity was observed in samples derived from familial breast cancer patients with $13964 \mathrm{C}$ variant that indicated the involvement of this variant in the TP53 gene overexpression. Based on these results, Lehman and et al considered $13964 \mathrm{C}$ variant as a specific germ-line mutation in familial breast cancer (Lehman et al., 2000).

In the years that followed, several other groups have been studied the importance of G13964C polymorphism in cancer predisposition and most of them were in contrast with Leman group's findings. In one study in the UK, on 19 patients with Li-Fraumeni and Li-Fraumeni like syndromes, the $\mathrm{C}$ allele was not found in any patient (Varley et al., 2001). In other studies on German and Australian women, $\mathrm{C}$ allele was found in both sporadic and familial breast cancer patients and there was no association between G13964C polymorphism and increased risk of cancer development (Marsh et al., 2001; Liu et al., 2004). Also, lack of association has been found in other case control studies, including HPV associated colon cancer in Turkish people and sporadic breast cancer in Kuwait and India (Buyru et al., 2005; Alawadi et al., 2011; Surekha et 
al., 2011). Recently, GC genotype has been reported to be involved in the increased risk of familial but not sporadic breast cancer in Tunisian women (Trifa et al., 2010).

In Iran, thyroid cancer is the 7th most prevalent cancer in females, 14th in the males and the 11th in both sexes, with the worst survival among Iranian-Azeri population (Akbari et al., 2008; Khayamzadeh et al., 2011). Exposure to ionizing radiation is one of the established risk factors for thyroid cancer (Duntas et al., 2006). This can be indicative of the fact that thyroid is a sensitive organ to tumor development induced by cellular stress and therefore, the status of This work has been financially supported by Azarbaijan Shahid Madani University under the grant number 401/461. gene, as a guardian of the genome against cellular stress, could be important in the thyroid cancer development and prognosis. Several studies have been shown that P53 mutations are mostly found in undifferentiated and poorly differentiated carcinoma and rarely attend in benign and differentiated tumors (Parameswaran et al., 2010; Montagna et al., 2011). However, besides mutations, This work has been financially supported by Azarbaijan Shahid Madani University under the grant number 401/461. has several polymorphisms that some of them could affect its function and be associated with increased risk of cancer. In this case-control study, for the first time, we have investigated the association of intron 6 G13964C substitution, a probable functional polymorphism of This work has been financially supported by Azarbaijan Shahid Madani University under the grant number 401/461., with the development of thyroid cancer (follicular adenoma and differentiated carcinoma). In our study, we could not find any association between G13964C polymorphism and the risk of thyroid cancer development among Iranian-Azeri population. Also, the $\mathrm{C}$ allele was not associated with clinicopathological characteristics, including stage, tumor type, lymph node metastasis, gender and age of diagnosis in thyroid patients.

Breast cancer is the first cause of cancer-related death and the first cancer to have been diagnosed in Iranian women (21.4\% of all cancers) (Babu et al., 2011). Therefore, it is crucial that we improve our understanding of this problem among Iranian women, both on the subject of genetic susceptibilities and environmental risk factors. Molecular changes, including the This work has been financially supported by Azarbaijan Shahid Madani University under the grant number 401/461. gene mutation and polymorphism, are related to increasing the risk of breast cancer development. This work has been financially supported by Azarbaijan Shahid Madani University under the grant number $401 / 461$. mutations occur in $15-30 \%$ of breast tumors that most of them lay in exons 4-9 (Olivier et al., 2001; Langerod et al., 2007). These mutations can occur as an early or late events and could be helpful as an prognostic factor in clinical practice (Olivier et al., 2006). Also, some of p53 polymorphisms may influence the susceptibility to breast cancer, however this relationship has not been confirmed in all of studies. In this study, we aimed to investigate whether the G13964C polymorphism is responsible for susceptibility to breast cancer in Iranian-Azeri patients. No association has been found between intron $6 \mathrm{G} 13964 \mathrm{C}$ polymorphism and the risk of breast cancer development as well as clinicopathological characteristics. The $\mathrm{C}$ allele frequency was even slightly less in patients rather than controls and like as many of the other similar investigations, G13964C substitution seemed to be a neutral polymorphism.

Based on our findings, we concluded that G13964C polymorphism lack a functional importance on anti-tumor activity of p53 and also is not in linkage disequilibrium with other susceptibility sites on p53. Therefore, G13964C polymorphism could not serve as a molecular marker for determining the risk of thyroid or breast cancers among Iranian-Azeri population.

\section{Acknowledgements}

The authors thank Imam Reza and Noor-E-Nejat hospitals staffs for their cooperation in sample and clinicopathological data collection procedure. This work has been financially supported by Azarbaijan Shahid Madani University under the grant number $401 / 461$.

\section{References}

Akbari M, Abachizadeh K, Khayamzadeh M, et al (2008). Iran cancer report. Cancer Research Center. Shahid Beheshti University of Medical Sciences. Tehran, Qom: Darolfekr. 101-6.

Akbari ME, Rafiee M, Khoei MA, et al (2011). Incidence and survival of cancers in the elderly population in Iran: 20012005. Asian Pac J Cancer Prev, 12, 3035-9.

Alawadi S, Ghabreau L, Alsaleh M, et al (2011). 53 gene polymorphisms and breast cancer risk in Arab women. Med Oncol, 28, 709-715.

Buller RE, Skilling JS, Kaliszewski S, et al (1995). Absence of significant germline p53 mutations in ovarian cancer patients. Gynecologic Oncology, 58, 368-74.

Buyru N, Tezol A, Dalay N (2005). p53 intronic G13964C variant in colon cancer and its association with HPV. Anticancer Res, 25, 2767-9.

Dehghan R, Hosseinpour Feizi MA, Pouladi N, et al (2014). Association of TP53 (-16ins-Pro) haplotype with the decreased risk of differentiated thyroid carcinoma in IranianAzeri Patients. Pathol Oncol Res, 20.

Duntas L, Grab-Duntas BM (2006). Risk and prognostic factors for differentiated thyroid cancer. Hell J Nucl Med, 9, 156-62.

Khayamzadeh M, Khayamzadeh M, Tadayon M, et al (2011). Survival of thyroid cancer and social determinants in Iran, 2001-2005. Asian Pac J Cancer Prev, 12, 95-98.

Langerod A, Zhao H, Borgan O, et al (2007). TP53 mutation status and gene expression profiles are powerful prognostic markers of breast cancer. Breast Cancer Res, 9, 30.

Legakis I, Syrigos K (2011). Recent advances in molecular diagnosis of thyroid cancer. J Thyroid res, 384213.

Lehman TA, Haffty BG, Carbone CJ, et al (2000). Elevated frequency and functional activity of a specific germ-line P53 intron mutation in familial breast cancer. Cancer Research, 60, 1062-9.

Liu X, Sinn HP, Ulmer HU, et al (2004). Intronic TP53 germline sequence variants modify the risk in german breast/ovarian cancer families. Hered Cancer Clin Pract, 2, 139-45.

Lowenstein D, Kasper D, Braunwald E, et al (2008). Harrison's principles of internal medicine. McGraw-hill Medical Publishing Division, New Delhi.

Machado-Silva A, Perrier S, Bourdon JC (2010). P53 family 
members in cancer diagnosis and treatment. Semin Cancer Biol, 20, 57-62.

Malkinson AM, You M (1994). The intronic structure of cancer-related genes regulates susceptibility to cancer. $\mathrm{Mol}$ Carcinog, 10, 61-5.

Marsh A, Spurdle AB, Turner BC, et al (2001). The intronic G13964C variant in p53 is not a high-risk mutation in familial breast cancer in Australia. Breast Cancer Research, 3, 346-9.

Montagna C, Di Cristofano A (2011). Thyrocyte-specific inactivation of P53 and Pten results in anaplastic thyroid carcinomas faithfully recapitulating human tumors. Oncotarget, 2, 1109-26.

Nikiforov YE, Biddinger PW, Thompson LDR (2009). Diagnostic pathology and molecular genetics of the thyroid. Baltimore, MD: Lippincott Williams and Wilkins, 94-102.

Olivier M, Goldgar DE, Sodha N, et al (2003). Li-Fraumeni and related syndromes: correlation between tumor type, family structure, and TP53 genotype. Cancer Res, 63, 6643-50.

Olivier M, Hainaut P (2001). TP53 mutation patterns in breast cancers: searching for clues of environmental carcinogenesis. Semin Cancer Biol, 11, 353-60.

Olivier M, Langerod A, Carrieri P, et al (2006). The clinical value of somatic TP53 gene mutations in 1,794 patients with breast cancer. Clin Cancer Res, 12, 1157-67.

Parameswaran R, Brooks S, Sadler GP (2010). Molecular pathogenesis of follicular cell derived thyroid cancers. Int J Surg, 8, 186-93.

Pouladi N, Kouhsari SM, Feizi MH, Gavgani RR, Azarfam P (2013). Overlapping region of p53/wrap53 transcripts: mutational analysis and sequence similarity with microRNA-4732- 5p. Asian Pac J Cancer Prev, 14, 3503-7.

Pouladi N, Kouhsari SM, Feizi MH, et al (2014). Lack of association of intron 316 bp polymorphism of TP53 with breast cancer among Iranian-Azeri patients. Asian Pac J Cancer Prev, 15, 2631-4.

Radha RK, P V, B K (2014). Histopathology and prognostic indices of carcinoma breast with special reference to p53 marker. J Clin Diagn Res, 7, 4-8.

Sedaie Bonab A, Pouladi N, Hosseinpourfeizi MA, et al (2014). Single-strand conformational polymorphism analysis of a common single nucleotide variation in WRAP53 gene, rs2287499, and evaluating its association in relation to breast cancer risk and prognosis among Iranian-Azeri population. Med Oncol, 31, 168

Shin MK, Kim JW (2014). Clinicopathologic and diagnostic significance of $\mathrm{p} 53$ protein expression in papillary thyroid carcinoma. Asian Pac J Cancer Prev, 15, 2341-4.

Sipos JA, Mazzaferri EL (2010). Thyroid cancer epidemiology and prognostic variables. Clinical Oncology, 22, 395-404.

Stegh AH (2012). Targeting the $P 53$ signaling pathway in cancer therapy - The promises, challenges, and perils. Expert Opin Ther Targets, 16, 67-83.

Surekha D, Sailaja K, Rao DN, et al (2011). Codon 72 and G13964C intron 6 polymorphisms of TP53 in relation to development and progression of breast cancer in India. Asian Pac J Cancer Prev, 12, 1893-8.

Trifa F, Karray-Chouayekh S, Mabrouk I, et al (2010). Haplotype analysis of P53 polymorphisms: Arg72Pro, Ins16bp and G13964C inTunisian patients with familial or sporadic breast cancer. Cancer Epidemiol, 34, 184-88.

Varley JM, McGown G, Thorncroft M, et al (2001). Significance of intron 6 sequence variations in the TP53 gene in LiFraumeni syndrome. Cancer Genet Cytogenet, 129, 85-7.

Walsh T, Casadei S, Coats KH, et al (2006). Spectrum of mutations in BRCA1, BRCA2, CHEK2, and TP53 in families at high risk of breast cancer. J Am Med Association, 